\title{
Validação da fita "CIMDER de 3 cores" como instrumento de detecção de risco nutricional entre pré-escolares*
}

\section{Validation of the three color CIMDER band, as an instrument of detection of nutritional risk in preschool}

\author{
Haroldo da S. Ferreira ${ }^{\star \star}$, Ari M.T. Ott**
}

FERREIRA, H. da S. \& OTT, A.M.T. Validação da fita "CIMDER de 3 cores" como instrumento de deteç̧ão de risco nutricional entre pré-escolares. Rev. Saúde Pública, 28:20-5, 1994. Analisou-se a validade da utilização da fita "CIMDER de 3 cores" pelos agentes de saúde do Estado de Rondônia, Brasil, como instrumento de deteç̧ão de risco nutricional entre crianças menores de 5 anos, para fins de encaminhamento para controle em unidades de maior complexidade que o posto de saúde. De uma amostra composta por 1.268 crianças, compararam-se os resultados da classificação nutricional obtida pela fita e da resultante da classificação de Gomez. A aplicação dos testes de validação resultou nos seguintes valores: sensibilidade $=77,1 \%$; especificidade $=68,8 \%$; valor preditivo positivo $=59,0 \%$; valor preditivo negativo $=83,7 \%$; taxa de falsos positivos $=31,2 \%$; taxa de falsos negativos $=22,9$. Excetuando a taxa de falsos positivos, os demais resultados foram considerados suficientemente satisfatórios para que se recomende o uso da fita CIMDER como instrumento de triagem pelos agentes de saúde de Rondônia. Outros indicadores mais específicos deverão ser adotados em nivel das unidades de saúde de maior complexidade para reduzir o número de falsos positivos nos programas de assistência dirigidos aos desnutridos.

Descritores: Circunferência braquial, instrumentação. Validez. Estado nutricional.

\section{Introdução}

A principal tarefa atribuida ao Núcleo de Pesquisas em Saúde ${ }^{* \star * *}$ (NUPES) foi a de elaborar e desenvolver o diagnóstico de saúde no Estado de Rondônia e, compreendido neste, o diagnóstico nutricional. Assim, já foi avaliado o estado nutricional dos pré-escolares ${ }^{5}$ e analisada a situação da prática do aleitamento materno ${ }^{4}$.

O sistema de saúde de Rondônia privilegia (dados de 1984) as ações a nível de assistência primária, adotando os princípios de hierarquização e regionalização, no intuito de assegurar à população amplo acesso aos serviços básicos de saúde,

* Trabalho realizado pelo Núcleo de Pesquisas em Saúde, órgão pertencente à Secretaria de Estado da Saúde de Rondônia, com recursos financeiros do Programa Integrado de Desenvolvimento do Noroeste do Brasil (POLONOROESTE).

** Departamento de Nutrição do Centro de Ciências da Saúde da Universidade Federal de Alagoas

*** Departamento de Ciências Biomédicas da Fundação Universidade Federal de Rondônia

Separatas/Reprints: H. da S. Ferreira - Rua Senador Teotônio Vilela, 75 - Bl/001 - Poço - 57030-640 - Maceió, AL. estruturados em ordem de complexidade crescente, iniciando-se pelo Posto de Saúde I, situado em área rural, contando com apenas um agente de saúde e que, portanto, representa a porta de entrada ao sistema para um grande contingente populacional ${ }^{11}$.

Considerando essas características, bem como a importância do controle do crescimento e desenvolvimento de crianças, dentro do elenco de ações que visam eliminar/minimizar os efeitos da desnutrição, sobretudo na faixa etária pré-escolar, planejou-se validar um instrumento simples e seguro que servisse como "screening" na deteç̧ão de casos de desvios do crescimento, para ser usado por agentes de saúde.

Dos métodos disponiveis, o que pareceu mais indicado aos objetivos propostos, foi a medida do perímetro braquial, por representar uma técnica de baixo custo, de fácil aplicação e bem aceito pela população.

O NUPES foi criado em 1983, vinculado à Secretaria do Estado de Saúde de Rondônia com o objetivo de operacionalizar o componente Pesquisa, integrante do Projeto de Saúde do Programa Integrado de desenvolvimento do Noroeste do Brasil (Polonoroeste) 
Embora mais difundida no Brasil, a fita de Shakir e Morley ${ }^{12}$, destinada a avaliar o estado nutricional através da medida do perímetro braquial, apresenta o inconveniente de, alegando-se que este é relativamente constante entre o primeiro e o quinto ano de vida, utilizar as mesmas dimensões para classificar crianças dentro dessa faixa etária, o que, obviamente, proporciona diferentes níveis de sensibilidade e especificidade conforme as diferentes idades. Apesar disso, a fita de Shakir e Morley pode ser de grande utilidade para trabalhos em localidades onde haja dificuldade na determinação precisa da idade dos indivíduos, a exemplo do inquérito realizado por Morais e col. ${ }^{10}$ entre crianças indias do Alto Xingu. Como isto não se aplica ao presente estudo, foi escolhida a fita proposta por Echeverri e col. ${ }^{3}$, do "Centro de Investigaciones Multidisciplinarias en Desarrolo Rural (CIMDER)", Colômbia, a qual apresenta medidas específicas para sete faixas etárias.

O objetivo do presente estudo foi analisar a validade da utilização, pelos agentes de saúde de Rondônia, da fita "CIMDER de três cores" como instrumento de triagem de crianças com risco nutricional, para encaminhamento aos centros de saúde, onde seriam melhor avaliadas e atendidas.

\section{Material e Método}

Foram estudadas 1.268 crianças de $0-60$ meses de idade, através da classificação de Gomez e da fita "CIMDER de 3 cores".

O plano amostral estabeleceu que as áreas urbanas de Ji-Paraná e Ariquemes e rural de Ouro Preto D'Oeste seriam auto-representativas, isto é, selecionadas com probabilidade 1. Para cada uma dessas áreas urbanas, decidiu-se estabelecer uma amostragem em dois estágios, com fração amostral total e probabilidade proporcional ao tamanho. Para as áreas urbanas, definiu-se a quadra (conjunto com no mínimo 70 e no máximo 110 domicílios), como unidade amostral de primeiro estágio e o domicílio como unidade de segundo estágio. Para a área rural, a unidade amostral de primeiro estágio foi a linha (estrada que, avançando mata adentro, permite o acesso e delimita cerca de 200 lotes); para unidade de segundo estágio tomou-se o lote.

Em função do número total de domicílios estimado para 1984 e da disponibilidade de recursos humanos e financeiros, decidiu-se tomar uma fração amostral total igual a $10 \%$ dos domicilios nas áreas urbanas de Ariquemes e Ji-Paraná. Para a área rural, levando-se em conta as características especiais do trabalho de campo em florestas, a fração amostral foi reduzida para 7,5\% dos lotes do Projeto Integrado de Colonização Ouro Preto. As quadras/domicilios e linhas/lotes foram selecionadas pelo sorteio casual para uma amostragem sistemática. No entanto, os problemas inerentes aos procedimentos de campo (recusas, domicílios fechados ou inalcançáveis e outros) reduziram a fração amostral para 8,2\% em Ariquemes, 8,3\% em Ji-Paraná e $5,2 \%$ em Ouro Preto D'Oeste, equivalendo, respectivamente, a 422,854 e 426 domicílios, num total de 1.702 .

Para cada domicílio sorteado, foram estudadas todas as crianças menores de cinco anos presentes no momento da entrevista. Os dados de peso e idade e, com a fita CIMDER, o perímetro braquial, eram coletados por entrevistadores previamente treinados e padronizados ${ }^{7}$. A tomada de peso foi efetuada por meio de balanças de gancho tipo "palanca", com capacidade para $20 \mathrm{~kg}$. Objetivando manter a qualidade dos dados, bem como evitar um possivel viés causado por discrepâncias entre entrevistadores, $1 / 6$ dos domicílios eram, após sorteio, revisitados pelo supervisor de campo a fim de verificar a informação inicialmente obtida.

A fita "CIMDER" classifica as crianças conforme uma escala de cores definida por diferentes pontos de corte estabelecidos para as seguintes faixas etárias, em meses: 0-3; 4-7; 8-11; 12-23; 24-47;

Tabela 1. Estrutura da tabela de contingência utilizada nos cálculos dos testes de validação.

\begin{tabular}{lccc}
\hline \multirow{2}{*}{ CIMDER } & $\begin{array}{c}\text { Gomez } \\
\text { (I, II e III graus) }\end{array}$ & Normal & Total \\
\cline { 2 - 5 } & $a$ & $b$ & $a+b$ \\
$\begin{array}{l}\text { Teste positivo } \\
\text { (amarelho) } \\
\text { vermelho) } \\
\begin{array}{l}\text { Teste negativo } \\
\text { (verde) }\end{array}\end{array}$ & $c$ & $d$ & $c+d$ \\
\hline Total & $a+c$ & $b+d$ & $a+b+c+d$
\end{tabular}


Tabela 2. Correspondência entre o estado nutricional classificado através do critério de Gomez e pelo perímetro braquial (CIMDER), relativo à crianças de 0-60 meses de idade do Estado de Rondônia, Brasil - 1985.

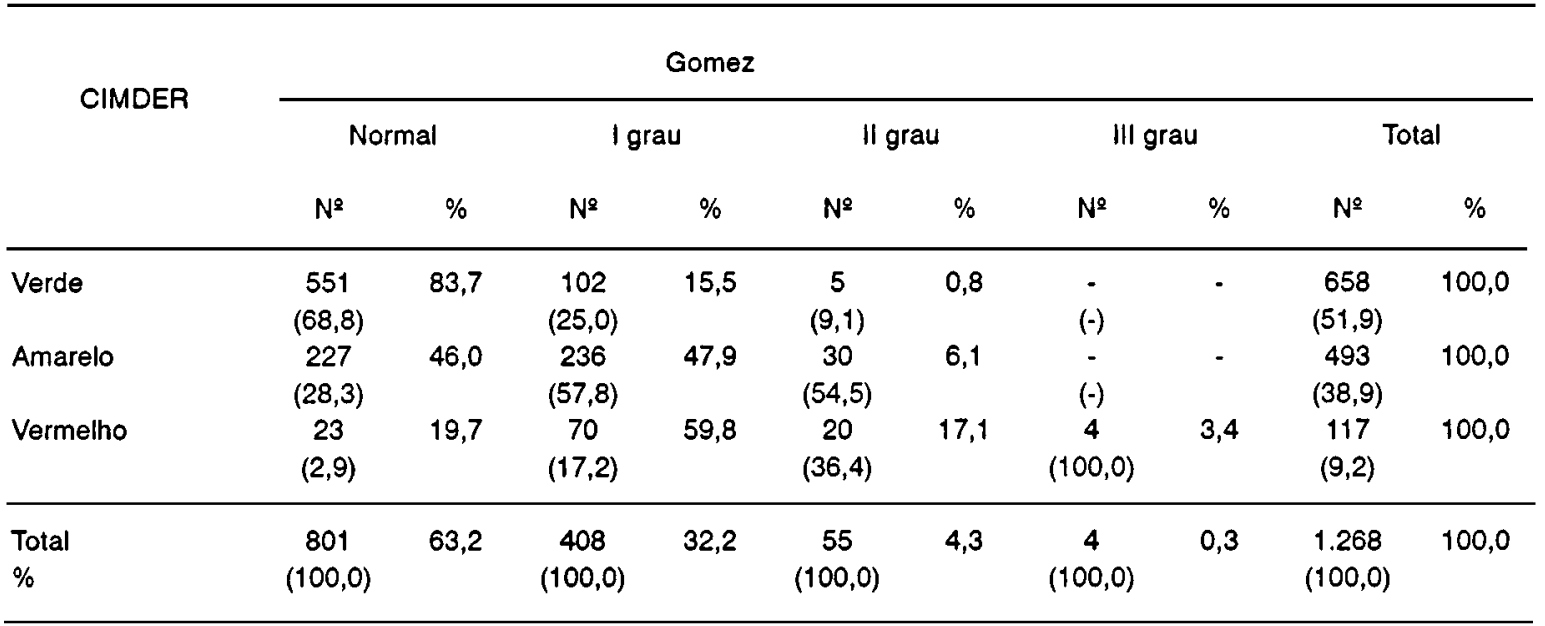

( ) = percentual referente à coluna

48-59 e, 60-71. As crianças classificadas no verde eram consideradas "normais"; no amarelo, como desnutridas leves; e, no vermelho, como portadoras de desnutrição de moderada a grave. $O$ treinamento dos entrevistadores para esta observação seguiu as recomendações do CIMDER ${ }^{3}$.

Para a validação da fita CIMDER, confrontamos os resultados obtidos através de sua classificação com os resultados encontrados pela classificação de Gomez. Para isto, montamos uma tabela de contingência onde se considerava desnutrida a criança que apresentasse adequação percentual para o indicador peso/idade inferior a $90 \%$ em relaçào à mediana do padrão antropométrico de referência do NCHS (National Center for Health Statistics) e, normais, as situadas acima desta adequação. As crianças classificadas no amarelo e no vermelho eram consideradas "teste positivo" e, quando no verde, como "teste negativo", conforme mostra a Tabela 1.

Calculou-se, então, a sensibilidade $(a / a+c .100)$, a especificidade $(\mathrm{d} / \mathrm{b}+\mathrm{d} .100)$, o valor preditivo positivo $(a / a+b .100)$, o valor preditivo negativo $(\mathrm{d} / \mathrm{c}+\mathrm{d} .100), \mathrm{a}$ taxa de falsos positivos $(\mathrm{b} / \mathrm{b}+\mathrm{d} .100) \mathrm{e}$ a taxa de falsos negativos $(\mathrm{c} / \mathrm{a}+\mathrm{c} .100)^{8}$.

A fim de se comparar os dados do presente estudo aos obtidos por Batista Filho e col. ${ }^{1}$, efetuouse, também, o procedimento empregado pelos cita- dos autores, os quais classificaram como desnutrição apenas os casos de II e III graus (adequação inferior a 75\%)e, como teste positivo, os casos classificados sob a condição vermelho da fita CIMDER.

\section{Resultados}

De acordo com a classificação de Gomez, das 1.268 crianças estudadas, $63,2 \%$ eram normais. Dos $36,8 \%$ casos de desnutrição, $32,2 \%$ eram de I grau, $4,3 \%$ de II grau e $0,3 \%$ de III grau.

$A$ avaliação nutricional através da fita CIMDER, classificou as crianças da seguinte forma: $51,9 \%$ normais, $38,9 \%$ com desnutrição leve e $9,2 \%$ com desnutrição de moderada a grave.

A Tabela 2 apresenta a correspondência entre os resultados dos dois métodos de classificação.

A Tabela 3 apresenta os valores de sensibilidade, especificidade, valor preditivo positivo, valor preditivo negativo, taxa de falsos positivos e taxa de falsos negativos, obtidos a partir dos dados de nossa amostra e dos divulgados por Batista Filho e col. ${ }^{1}$ e pelo CIMDER ${ }^{3}$.

\section{Discussão}

Um dos grandes problemas na definição de pontos de corte em sistemas de classificação nutri- 
Tabela 3. Resultado dos testes obtidos a partir da classificação do estado nutricional pela medida do perímetro braquial (fita CIMDER) em comparação à classificação de Gomez em crianças menores de 5 anos do Estado de Rondônia (Ferreira) e das amostras de Batista Filho (1992) e do CIMDER.

\begin{tabular}{lcccc}
\hline Teste & Ferreira* & Ferreira** & Batista Filho** & CIMDER* \\
\hline Sensibilidade & 77,1 & 40,7 & 63,5 & 93,9 \\
Especificidade & 68,8 & 92,3 & 87,9 & 63,2 \\
Valor preditivo positivo & 59,0 & 20,5 & 40,0 & 83,3 \\
Valor preditivo negativo & 83,7 & 96,9 & 95,0 & 84,3 \\
Taxa de falsos positivos & 31,2 & 7,7 & 12,1 & 36,7 \\
Taxa de falsos negativos & 22,9 & 59,3 & 36,5 & 6,0 \\
\hline
\end{tabular}

Fonte:

Ferreira, H. da S. - Dados inéditos

Batista Filho e col. ${ }^{1}(1992)$

Echeverri e col. ${ }^{3}$ (1979) - (CIMDER)

* Tabela de contingência montada a partir dos seguintes dados:

$a=$ desnutridos de I, II, e III graus (Gomez) + amarelho/vermelho (CIMDER).

$b=$ normais (Gomez) + amarelo/vermelho (CIMDER).

$c=$ desnutridos de l, ll e lll graus (Gomez) + verde (CIMDER).

$d=$ normais (Gomez) + verde (CIMDER).

* Tabela de contingência montada a partir dos seguintes dados:

$a$ = desnutridos de II e III graus (Gomez) + vermelho (CIMDER)

$b=$ normais e desnutridos de I grau (Gomez) + vermelho (CIMDER).

c = desnutridos de $\|$ e III graus (Gomez) + verde/amarelo (CIMDER).

$d$ = normais e desnutridos de I grau (Gomez) + verde/amarelo (CIMDER).

cional (ou em qualquer outro sistema de classificação) é o fato de que as variáveis sensibilidade e especificidade são inversamente proporcionais, ou seja, quando se privilegia a primeira, detecta-se um maior contingente de desnutridos dentro da amostra estudada. No entanto, será grande o número de falsos positivos. Para se reduzir esse inconveniente, é preciso que o indicador ganhe em especificidade, o que pode aumentar o número de falsos negativos e, assim, deixar de fora da triagem grande quantidade de indivíduos realmente portadores da desnutrição.

Para contornar esta dificuldade, Monteiro e col. $^{9}$ propuseram pontos de corte para a medida do perimetro braquial que apresentassem sensibilidade e especificidade semelhantes. Segundo dados dos citados autores, "para cada faixa anual de idade, haveria um determinado nivel do perimetro braquial que separaria, em $75-80 \%$ das vezes cortetamente, os pré-escolares desnutridos dos demais", sendo que, para os casos moderados e graves, a detecção seria de $95 \%$.

A utilização de pontos de corte onde a sensibilidade e a especificidade da classificação tendam a se igualar, parece bastante útil para o diagnóstico nutricional de populações, de vez que, conforme relata Monteiro e col. ${ }^{9}$, "os erros cometidos ao se avaliar desnutridos e eutróficos tenderiam a se anular, redundando em prevalência global da desnutrição semelhante a que seria obtida pela classificação de referência". No entanto, quando o objetivo da classificação não é tão somente de determinação de prevalência, mas também triagem e/ou controle, deve-se privilegiar a sensibilidade ou a especificidade, respectivamente.

No presente caso, o interesse foi validar um instrumento simples, prático, acessivel aos agentes de saúde de Rondônia, os quais não dispõem de grandes habilidades e infra-estrutura para utilização de métodos mais sofisticados na deteç̧ão e triagem de casos de desnutrição no âmbito de sua área de cobertura, o que nos inclina a privilegiar a sensibilidade. Embora os pontos criticos, propostos por Monteiro e col. ${ }^{9}$, propiciaram valores de sensibilidade e especificidade um pouco melhores do que os obtidos no presente estudo, "é possível que em populações que se afastem muito das condições nutricionais da população que estudamos, não se 
mantenha a correspondência entre a adequação ponderal e o perímetro braquial. Nesta situação, poderiam ser outros os pontos críticos que melhor identificariam a fronteira entre a desnutrição e a eutrofia (Monteiro e col. ${ }^{9}$ ).

$\mathrm{Na}$ Tabela 3, as diferenças observadas entre os resultados (Tabela 3 - coluna Ferreira ${ }^{\star \star}$ ) e os divulgados por Batista Filho e col. ${ }^{1}$ (Tabela 3), justificam-se em virtude das diferenças em termos de prevalência e magnitude (graus) da desnutrição das respectivas amostras, pois a sensibilidade tende a aumentar quando a prevalência das formas moderada e grave são maiores, o que ocorreu na amostra de Batista Filho e col. ${ }^{1}$ em virtude da inclusão de casos hospitalares. No estudo realizado pelo CIM$\mathrm{DER}^{3}$, a sensibilidade da fita variou em amostras formadas por individuos com desnutrição de I, II e III graus para $92,2 \%, 97,1 \%$ e $100,0 \%$, respectivamente.

Embora Batista Filho e col. ${ }^{1}$ admitam como satisfatórios os valores obtidos para sensibilidade $e$ especificidade, consideram o valor preditivo positivo $(40,0 \%)$ bastante baixo para recomendar o uso da fita, nos pontos de corte propostos. No presente estudo (Tabela 3 - coluna Ferreira ${ }^{*}$ ) em que se consideraram todos os casos de desnutrição, e não apenas os graus II e III e aceitando como diagnóstico correto, a condição amarelo e vermelho da fita, o valor preditivo positivo foi de $59,0 \%$, muito embora a taxa de falsos positivos tenha se elevado para
$31,2 \%$.

Todos os trabalhos de validação do uso da medida do perímetro braquial, aqui citados, apresentam falha em comum com os presentes dados: a utilização da classificação de Gomez como "gold standard". Essa classificação constitui um dos mais antigos sistemas de avaliação do estado nutricional. $\mathrm{Na}$ seleção dos pontos de corte, Gomez levou em consideração as caracteristicas de peso em relação à idade de crianças internadas com desnutrição severa. Segundo Gibson ${ }^{6}$, o método não possibilita diferenciação entre kwashiorkor e marasmo, nem entre nanismo e emaciação pelo fato de que a estatura não é levada em consideração. Desse modo, crianças com baixo peso para a idade podem representar diferentes situaçōes nutricionais, tais como nanismo (desnutrição pregressa), emaciação (desnutrição aguda) ou nanismo + emaciação (desnutrição crônica), portanto, não estima a duração do episódio. Outra desvantagem surge em virtude da possibilidade de edema. Assim, os resultados obtidos a partir da classificação de Gomez não representam bom padrão para validação de outros métodos, em virtude de suas próprias limitações e pela existência de metodologias mais satisfatórias, como as discutidas por Batista Filho e $\mathrm{col}^{2}{ }^{2}$, baseadas na utilização de percentis ou desvios-padrões nas relações peso/idade, altura/idade e peso/altura. Sugerese, então, a continuidade desses estudos utilizandose tais metodologias, o que, no entanto, não destitui

Tabela 4. Comparação entre os pontos de corte da classificação de Gomez (déficits percentuais) e os determinados pelos percentis e desvios-padrōes em diferentes idades da relação peso/idade de meninos menores de 5 anos do padrão do NCHS (National Center for Health Statistics).

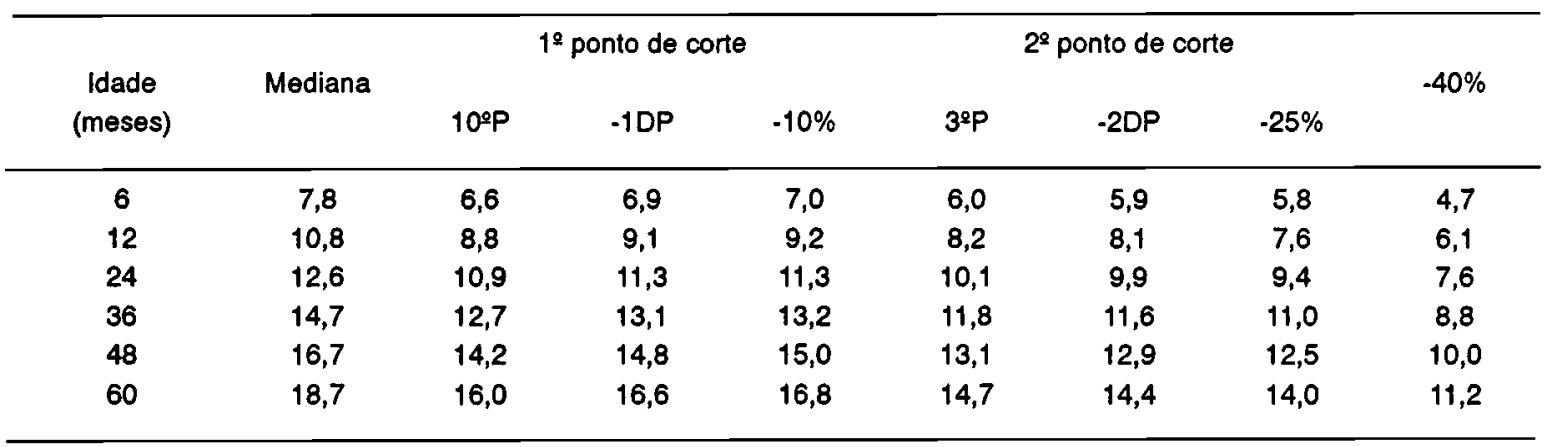

$P=$ Percentil

DP = desvio-padrão 
o valor dos dados até agora divulgados, de vez que a classificação de Gomez, apesar de seus inquestionáveis óbices, apresenta, em seus pontos de corte, razoável concordância com os níveis percentilares propostos para separação entre desnutridos e eutróficos, concordància que é ainda mais expressiva, quando os pontos de corte são definidos através de desviospadröes da mediana, conforme mostra a Tabela 4.

Diante do exposto, concluimos pela validade da utilização da fita CIMDER de três cores pelos agentes de saúde em virtude de julgarmos satisfatórios para os objetivos propostos, os valores obtidos nos testes de validação, exceto para a taxa de falsos positivos $(31,2 \%)$, porem, sendo a fita CIMDER adotada apenas como um "screening" de primeiro nível, a adoção de outros indicadores, mais específicos, em nível de centros de saúde, reduzirá a probabilidade de inclusão de falsos positivos nos programas de controle.

\section{Agradecimentos}

Ao Prof. Odécio Sanches, da Escola de Enfermagem de Ribeirão Preto, por sua assessoria na definição do plano amostral.

FERREIRA, H.da S. \& OTT, A.M.T. [Validation of the three color CIMDER band, as an instrument of detection of nutritional risk in preschool]. Rev. Saúde Pública, 28:20-5, 1994. The value of the use, by the health agents in Rondonia, Brazil, of the nutritional classification proposed by the Multidisciplinary Research Center for Rural Development (CIMDER), Colombia, known as the three color CIMDER band, is analyzed. The band, used to measure arm circumference, would be used as an instrument for the detection of nutritional risk in children under five years of age and for refering them to larger, more complex, health units. For this purpose, a sample of 1,268 children were studied. The results of the nutritional classification obtained by the band and the results of Gomez classification were compared. The application of the validations tests resulted in the following values: sensibility $=77.1 \%$; specificity $=$ $68.8 \%$; positive predictive value $=59.0 \%$; negative predictive value $=83.7 \%$; rate of false positives $=31.2 \%$ and rate of false negatives $=22.9 \%$. Except for the rate of false positives, the rest of the results were considered to be satisfactory, sufficiently so to recommend the use of the CIMDER band as an instrument of selection by the health agents in Rondônia. More specific indicators should be adopted at the larger, more complex health units, with a view to reducing the number of false positives in the programs for attendance to the undernourished.

\section{Referências Bibliográficas}

1. BATISTA FILHO, M.; NACUL, L.C.; BEZERRA, T.C.A.; FERNANDES, A. S. Validação da cinta "CIMDER" como método de avaliação do estado nutricional de crianças . Rev. Inst. Materno - Infant. Pernambuco, 6:15-21, 1992.

2. BATISTA FILHO, M.; COUTINHO, D.C.; RIBEIRO, M.A. O risco de desnutrição "atual" em crianças brasileiras situadas abaixo do percentil 10 da relação peso/idade. Rev. Inst. Materno - Infant. Pernambuco, 6:99-106,1992.

3. ECHEVERRI, O.; DOENHEM, H.; VILLAFANE, P. Validación de un instrumento para medir el estado nutricinal en niños de 0-6 anos. Cali, Centro de Investigaciones Multidisciplinárias en Desarrolo Rural, 1979.

4. FERREIRA, H. da S. \& OTT, A.M.T. Situação do aleitamento materno em três municípios do estado de Rondônia, Brasil, 1985. In: Congresso Brasileiro de Nutrição, 11², Salvador, 1987. Anais, Salvador, 1987, p. 66.

5. FERREIRA, H. da S. \& OTT, A.M.T. Avaliação do estado nutricional de crianças menores de 5 anos do estado de Rondônia, Brasil. Rev. Saúde Pública, 22:179-83, 1988.

6. GIBSON, R.S. Evaluation of anthropometric indices. In: Gibson, R.S. Principles of nutritional assessment. New York, Oxford University Press, 1990. p. 247-60.

7. GOUVEIA, E.L.C. Diagnóstico do estado nutricional da população. In: Chaves, N. Nutrição básica e aplicada. Rio de Janeiro, Editora Guanabara Koogan, 1978. p. 245-74.

8. HENNEKENS, C.H. \& BURING, J.E. Screening. In: Hennekenns, C. Epidemiology in medicine. Boston, Sherry L. Mayrent Ed., 1987. p. 327-45.

9. MONTEIRO, C.A.; BENICIO, M.H.D.; GANDRA, Y.R. Uso da medida do perimetro braquial na detecção do estado nutricional do pré-escolar. Rev. Saúde Pública, 15(supl): 48-63, 1981

10. MORAIS, M.B. de; FAGUNDES NETO, U.; BARUZZI, R.G.; PRADO, M.C.O.; WEHBA, J.; SILVESTRINI, W.S. Estado nutricional de crianças indias do Alto Xingu e avaliação do uso do perimetro braquial no diagnóstico da desnutrição protéico-calórica. Rev. Paul. Med, 108:245-51, 1990.

11. OTT, A.M.T.; COIMBRA JR, C.E.A.; FERREIRA, H. da S.; SMANIO NETO, L.; BRANCO, M.P.S. Diagnóstico das condiçōes de saúde em Rondônia: projeto de pesquisa. Secretaria de Estado de Saúde de Rondônia/CNPa/Banco Mundial, Rondônia , 1984, [mimiografado].

12. SHAKIR, A. \& MORLEY, D. Measuring malnutrition Lancet, 1:758-9, 1974
Recebeido para publicação em 16.8 .1993 Reapresentado em 8.11.1993 Aprovado para publicação em 5.1.1994 